\title{
Correction to: Predation capacity of phytoseiid mites (Mesostigmata: Phytoseiidae) from Brazil on eggs of Diaphorina citri (Hemiptera: Liviidae)
}

\author{
Sofía Jiménez Jorge • D. Rueda-Ramírez • G. J. de Moraes
}

Published online: 28 April 2021

(C) Springer Nature B.V. 2021

\section{Correction to: Phytoparasitica}

https://doi.org/10.1007/s12600-021-00898-9

The original version of this article unfortunately contained an incorrect presentation of author name. S. Jiménez Jorge should be Sofía Jiménez Jorge.

Publisher's note Springer Nature remains neutral with regard to jurisdictional claims in published maps and institutional affiliations.

The online version of the original article can be found at https://doi.org/10.1007/s12600-021-00898-9

\section{S. J. Jorge $(\bowtie)$}

Depto. de Fitossanidade, FCAV-UNESP, 144884-900

Jaboticabal, São Paulo, Brazil

e-mail: saposoa40_20@hotmail.com

S. J. Jorge

Asociación de Productores de cítricos del Perú (PROCITRUS),

Lima, Peru

D. Rueda-Ramírez

Depto. De Agronomía, Facultad de Ciencias Agrarias,

Universidad Nacional de Colombia, Carrera 30 No. 45-03,

Edificio, 500 Bogotá, Colombia

\section{G. J. de Moraes}

CNPq researcher, Depto. de Entomologia e Acarologia, ESALQ Universidade de São Paulo, 13418-900 Piracicaba, São Paulo, Brazil 